\title{
Vacuum-fluctuation-induced Dephasing of a Qubit in Circuit Quantum Electrodynamics
}

\author{
Y.-W. Kim ${ }^{1}$, K.-H. Lee ${ }^{1,2}$, Kicheon Kang ${ }^{1,3 *}$ \\ ${ }^{1}$ Department of Physics, Chonnam National University, Gwangju 500-757, Korea \\ ${ }^{2}$ Department of Physics, Ben Gurion University of the Negev, Beersheba 84105, Israel \\ ${ }^{3}$ Asia Pacific Center for Theoretical Physics, POSTECH, Gyeongbuk 790-784, Korea
}

\begin{abstract}
We investigate the measurement-induced dephasing of a qubit coupled with a single-mode cavity in the vacuum limit. Dephasing of the qubit state takes place through the entanglement of the qubit and the single probe photon sent to the cavity, while the cavity mode never occupies a photon. We find that the qubit state is dephased even if the cavity is always in the vacuum state. This dephasing is caused purely by the interaction between the qubit and the vacuum field. We also show that this vacuum-fluctuation-induced dephasing takes place much faster than the spontaneous decay of the qubit excited state, and therefore our prediction is observable in a real experiment.
\end{abstract}

Introduction - The complementarity principle ${ }^{1)}$ is the basis of quantum physics. It demands that a possibility of extracting the state information of a "system" with an "apparatus" inevitably causes a reduction in the quantum interference of the state being monitored. This so-called "measurement-induced dephasing" has been clearly confirmed in real experiments with photons ${ }^{2)}$ Rydberg atoms, ${ }^{3)}$ and electrons in solid state interferometers. ${ }^{4,5)}$ Circuit (cavity) quantum electrodynamics (QED) architecture ${ }^{6-8)}$ is an ideal playground for studying complementarity and measurement-induced dephasing. The dispersive measurement scheme provides a non-invasive readout suitable for quantum information processing, and is also ideal for testing the complementarity principle. In this system, it is generally believed that the measurement-induced dephasing is caused by the photon number fluctuations in the cavity, ${ }^{6,9-13)}$ as it has been experimentally demonstrated. ${ }^{14,15)}$

In contrast to this semiclassical photon shot-noise dephasing, here we propose that measurement-induced dephasing takes place even in the vacuum limit of the cavity where the photon number fluctuation is completely suppressed. To be specific, we consider a qubit coupled to a resonator with a single photon mode in the dispersive limit. We show that the qubit is dephased due to the vacuum field in the resonator with the condition that the photon number is always zero in the cavity. This demonstrates a novel property of the vacuum manifested in the context of the quantum mechanical complementarity.

Model Hamiltonian - A qubit interacting with a singlemode resonator is represented by the Hamiltonian,

$$
H=H_{0}+H_{c}+V_{0 c},
$$

where $H_{0}$ denotes the qubit+cavity mode represented by the usual Jaynes-Cummings model ${ }^{16)}$ with rotating wave approximation. $H_{c}$ and $V_{0 c}$ represent the continuum modes outside the cavity and the cavity-continuum interaction, respectively:

$$
H_{0}=\hbar \omega_{r}\left(a^{\dagger} a+\frac{1}{2}\right)+\frac{\hbar \omega_{q}}{2} \hat{\sigma}_{z}+\hbar g\left(a^{\dagger} \sigma^{-}+\sigma^{+} a\right)(1 \mathrm{~b})
$$

$$
\begin{aligned}
H_{c} & =\sum_{k} \hbar v_{k}\left(a_{k}^{\dagger} a_{k}+\frac{1}{2}\right), \\
V_{0 c} & =\sum_{k} \hbar \alpha_{k}\left(a_{k}^{\dagger} a+a^{\dagger} a_{k}\right) .
\end{aligned}
$$

The parameters $\omega_{r}, \omega_{q}, g, v_{k}$, and $\alpha_{k}$ denote the resonance frequency of the cavity, qubit frequency, the coupling between the qubit and the cavity, the continuum mode frequency, and the coupling between the cavity and the continuum, respectively. There are two important parameters governing the characteristics of the system in our context: the detuning of the qubit and the cavity frequencies, $\Delta \equiv \omega_{q}-\omega_{r}$, and the decay rate of the cavity mode, $\Gamma \equiv 2 \pi \sum_{k}\left|\alpha_{k}\right|^{2} \delta\left(\omega_{r}-v_{k}\right)$, assumed to be independent of the frequency. In the dispersive limit $(|\Delta| \gg g), H_{0}$ is transformed to an effective Hamiltonian,

$$
\tilde{H}_{0}=\hbar \omega_{r}\left(a^{\dagger} a+\frac{1}{2}\right)+\frac{\hbar}{2}\left[\omega_{q}+2 \chi\left(a^{\dagger} a+\frac{1}{2}\right)\right] \hat{\sigma}_{z},
$$

where $\chi=g^{2} / \Delta$ is the dispersive shift.

Measurement-induced dephasing in the vacuum limit of the resonator - The measurement-induced dephasing is generally regarded as a result of the photon number fluctuations. For instance, in a theory based on the continuous weak measurement $(\chi \ll \Gamma),{ }^{6,9)}$ the phase factor of the qubit decays as $\left\langle e^{i \varphi(t)}\right\rangle=\exp \left(-\Gamma_{\phi} t\right)$, with the dephasing rate

$$
\Gamma_{\phi}=2 \theta_{0}^{2} \Gamma \bar{n},
$$

where $\theta_{0} \equiv 2 \chi / \Gamma$ and $\bar{n}$ is the average photon number in the cavity. This property of dephasing rate, which is governed by the photon number fluctuation, has been confirmed in experiments. ${ }^{14,15)}$ The photon shot noise dephasing is verified also in the strong measurement limit where single photons fully dephase the qubit. ${ }^{13)}$ Both in the weak and in the strong measurement limits, the photon number fluctuation in the cavity has been regarded as the source of the measurement-induced dephasing. In these studies, the effect of the vacuum field was not taken into account, and the dephasing rate vanishes in the vacuum limit.

We investigate the role of the vacuum fluctuation in the measurement-induced dephasing. Our aim is to show that $\Gamma_{\phi}$ 
does not vanish even when the cavity is always in the vacuum state. Let us consider a measurement setup (see Fig. 1) where single "probe" photons are sent to a cavity interacting with a qubit. We consider a circuit QED system for a possible realization, but a cavity QED setup can also be used. The key point is that, during its travel through the cavity, the outcome of a probe photon becomes entangled with the qubit state, and thereby produces a "measurement-induced dephasing" of the qubit.

The initial state of the qubit-probe photon composite,

$$
|\psi(0)\rangle=\left(c_{g}|g\rangle+c_{e}|e\rangle\right) \otimes|D\rangle,
$$

evolves into

$$
|\psi(t)\rangle=c_{g}|g\rangle \otimes\left|D_{g}(t)\right\rangle+c_{e}|e\rangle \otimes\left|D_{e}(t)\right\rangle .
$$

The time evolution of a probe photon state, $\left|D_{s}\right\rangle$, depends on the qubit state $s(=g$ or $e$ ), and is given by

$$
\left|D_{s}(t)\right\rangle=A_{s}(t)|1,0\rangle+\int B_{s}(k, t)\left|0,1_{k}\right\rangle d k,
$$

where the photon state is given in the number state representation $\left|n, n_{k}\right\rangle$ with $n$ and $n_{k}$ being the photon numbers in the cavity and in the mode $k$ of continuum, respectively.

First, we consider a simple case of injecting a monochromatic single photon, $\left|0,1_{k_{0}}\right\rangle$. This case is specified by the initial condition,

$$
A_{s}(0)=0, \quad B_{s}(k, 0)=\delta\left(k-k_{0}\right) .
$$

The time evolutions of the coefficients $A_{s}(t)$ and $B_{s}(k, t)$ are found to be

$$
\begin{aligned}
A_{s}(t) & =\frac{\alpha_{k_{0}}}{\omega_{s}-v_{k_{0}}-i \Gamma / 2}\left(e^{-\Gamma t / 2}-e^{i\left(\omega_{s}-v_{k_{0}}\right) t}\right), \\
B_{s}(k, t) & =\delta\left(k-k_{0}\right)+\frac{\alpha_{k}^{*} \alpha_{k_{0}}}{\omega_{s}-v_{k_{0}}-i \Gamma / 2} \\
& \times\left(\frac{e^{-i\left(\omega_{s}-v_{k}-i \Gamma / 2\right) t}-1}{\omega_{s}-v_{k}-i \Gamma / 2}-\frac{e^{-i\left(v_{k_{0}}-v_{k}\right) t}-1}{v_{k_{0}}-v_{k}}\right),
\end{aligned}
$$

where $\omega_{s}=\omega_{r} \pm \chi$ (for $s=e / g$ ) is the qubit-dependent cavity frequency shifted by the dispersive interaction. The qubit state information is eventually encoded in the asymptotic limit of the photon state $\left|D_{s}(t \rightarrow \infty)\right\rangle$. This asymptotic limit, obtained by taking $v_{k} \rightarrow v_{k}+i \epsilon$ and $t \rightarrow \infty$, is given by

$$
\left|D_{s}(\infty)\right\rangle=\frac{\omega_{s}-v_{k_{0}}}{\omega_{s}-v_{k_{0}}-i \Gamma / 2}\left|0, k_{0}\right\rangle+\frac{i \Gamma / 2}{\omega_{s}-v_{k_{0}}-i \Gamma / 2}\left|0,-k_{0}\right\rangle .
$$

The coherence factor, defined as

$$
\lambda \equiv\left\langle D_{e}(\infty) \mid D_{g}(\infty)\right\rangle,
$$

is a measure of interference between the two qubit states. For calculating $\lambda$, we use the condition $v_{k 0}=\omega_{g}$, that is, the input frequency is in resonance with the dressed cavity frequency when the qubit is in the ground state. In fact, this is an optimal measurement condition, and we find

$$
\lambda=\frac{i \Gamma / 2}{2 \chi+i \Gamma / 2} \text {. }
$$

In the strong measurement limit $(\chi \gg \Gamma), \lambda \approx 0$. This implies that the qubit state is fully dephased when a single photon is sent to the cavity. It should be noted that this dephasing has nothing to do with the photon number fluctuation, but origi- nates purely from the vacuum field in the cavity. To confirm this statement, we have to check the occupation probability of a photon in the cavity at an arbitrary time $t, P_{1}(t)$, which is given by

$$
P_{1}(t)=\left|A_{s}(t)\right|^{2},
$$

in our case. As one can find from Eq. (7a), $A_{s}(t) \sim \alpha_{k_{0}} / \Gamma$. Note that $\Gamma=2 \pi|\alpha|^{2} \rho$, where $\rho$, the density of states at the input frequency $v_{k 0}$, is a macroscopic quantity and is proportional to $M$, the number of modes in the reservoir which are coupled to the cavity. Therefore, $\alpha_{k 0} \propto 1 / \sqrt{M}$, and we find that $P_{1}(t)$ is proportional to $1 / M$. Since $M \rightarrow \infty$ for a reservoir with continuous spectrum, $P_{1}(t)=0$. This implies that photon number fluctuation is absent in the cavity, since a photon never occupies the cavity mode during the interaction process. The full dephasing of the qubit in the strong measurement limit is understood only in terms of the interaction between the qubit and the vacuum field, which transcends the semiclassical concept of dephasing generated by the photon number fluctuation.

The above discussion with a monochromatic probe photon already includes the essence of the vacuum-fluctuationinduced dephasing. A drawback of this treatment is that the dephasing time is infinity in this ideal case, because the asymptotic limit cannot be reached in a finite time interval with a monochromatic wave. Next, we treat a more realistic case of sending a single photon with its frequency dispersion $\Delta v$. This can be modeled, for example, by an input of the Gaussian wave packet:

$$
\left|D_{s}(t=0)\right\rangle=N_{0} \int e^{-\left(v_{k}-v_{k_{0}}\right)^{2} /(2 \Delta v)^{2}}\left|0,1_{k}\right\rangle d v_{k} .
$$

The time evolution of this state is given by $\left|D_{s}(t)\right\rangle=$ $\exp (-i H t)\left|D_{s}(0)\right\rangle$, and calculating the coherence factor (Eq. (9)) from the asymptotic limit of $\left|D_{s}(t)\right\rangle$ is straightforward. We find

$$
\lambda=\lambda\left(\frac{2 \Delta v}{\Gamma}, \frac{2 \chi}{\Gamma}\right),
$$

where the function $\lambda(x, y)$ is given by

$$
\begin{aligned}
\lambda(x, y)= & -\sqrt{\frac{\pi}{2}} \frac{y}{x(y+i)}\left[e^{(1-i 2 y)^{2} / 2 x^{2}} \operatorname{erfc}\left(\frac{1-i 2 y}{\sqrt{2} x}\right)\right. \\
& \left.+e^{1 / 2 x^{2}} \operatorname{erfc}\left(\frac{1}{\sqrt{2} x}\right)\right] .
\end{aligned}
$$

The probe photon reaches the asymptotic limit more rapidly as the pulse length $(\Delta x)$ is reduced, or equivalently, as $\Delta v$ increases (see Fig. 2). This implies that a measurement can be performed faster for a larger dispersion in the input frequency. For a pulse that is too short, however, the probe photon cannot really detect the qubit state $(|\lambda| \sim 1)$ because of the large frequency dispersion $\Delta v$ that obscures the resolution in the measurement. This is verified in the behavior of $|\lambda|$ as a function of $\Delta v$ (Fig. 2(b)). The optimal measurement can be obtained by imposing the condition $|\lambda|=1 / e$. The dephasing time is determined by the travel time of the input wave through the cavity that satisfies the condition $|\lambda|=1 / e$. In the strong measurement limit, $\chi \gg \Gamma$, we find that the vacuuminduced dephasing rate, $\Gamma_{\phi}$, is proportional to the cavity decay 
rate $\Gamma$ as

$$
\Gamma_{\phi} \simeq 1.05 \Gamma .
$$

Our discussion of the dephasing rate can be easily extended to the case where the cavity has $n$ photons. In this case, the effective coupling of the cavity and the reservoir is enhanced by the factor of $n+1$ compared to the vacuum limit, and thus the dephasing rate is also enhanced by the same factor as

$$
\Gamma_{\phi}(n)=(n+1) \Gamma_{\phi} .
$$

The vacuum-fluctuation-induced dephasing takes place not only in the strong measurement limit, but also in the weak measurement limit $(\chi \ll \Gamma)$. In this case, the single probe photon only has a negligible effect on the qubit state. Instead, the qubit state can be dephased by a continuous injection of probe photons. The interference of the qubit state is reduced by the factor $\left|\lambda^{m}\right|$ in this case, which can be written as

$$
\left|\lambda^{m}\right| \simeq e^{-\Gamma_{\phi} m \delta t},
$$

where $m(\gg 1)$ and $\delta t$ are the number of injected photons and the time interval between the successive transmission of the input packets, respectively. $\lambda$ is the coherence factor due to a single probe photon, given by Eq.(13). In general, the vacuum-fluctuation-induced dephasing rate, $\Gamma_{\phi}$, depends on the packet width of the input photons. An optimal setting of measurement is the case where the frequency dispersion $(\Delta v)$ of the input wave is comparable to the cavity-reservoir coupling $(\Gamma)$. We find that, for $\Delta v=\Gamma$,

$$
\Gamma_{\phi}=\beta \frac{(2 \chi)^{2}}{\Gamma},
$$

with the constant $\beta=2(3-2 e \sqrt{\pi} \operatorname{erfc}[1])=2.97$.

The behavior of the vacuum-fluctuation-induced dephasing rate is summarized in Fig. 4. In the strong measurement limit, $\Gamma_{\phi}$ is determined from the condition $|\lambda|=1 / e$ with $\lambda$ given by Eq. (13). The result for a continuous weak measurement limit is obtained from Eq. (17).

A necessary condition for an experimental observation of the vacuum-field-induced dephasing is that the dephasing should take place before the spontaneous relaxation of the qubit state. That is, the dephasing rate should be larger than the rate of spontaneous relaxation. Here we show that this is indeed the case in both the strong and the continuous weak measurement limits. The spontaneous decay rate in our system, denoted by $\gamma$, is governed by the Purcell effect, ${ }^{17)}$ and is given as

$$
\gamma=2 g^{2} \frac{\Gamma / 2}{\Delta^{2}+(\Gamma / 2)^{2}} .
$$

We consider only the dispersive limit in which the detuning $\Delta$ is greater than the coupling strengths $g$ and $\Gamma$. In this limit, $\gamma$ is reduced to

$$
\gamma \simeq\left(\frac{g}{\Delta}\right)^{2} \Gamma .
$$

Comparing Eq. (14) and Eq. (19), we find that $\Gamma_{\phi} / \gamma \simeq(\Delta / g)^{2}$ in the strong measurement limit $(\chi \gg \Gamma)$. That is, the dephasing takes place faster than the spontaneous decay by the factor $(\Delta / g)^{2}$. In the case of continuous weak measurement, the ratio is given by $\Gamma_{\phi} / \gamma \simeq 12(g / \Gamma)^{2}$ (compare Eq.(17) and Eq.(19)), implying that the dephasing can be observed in the strong coupling regime $(g \gg \Gamma)$. Therefore, in any case, our prediction of vacuum-induced dephasing is observable before the qubit is spontaneously relaxed to its ground state.

Finally, we discuss in more detail the meaning of cavity vacuum during the interaction between the qubit and the probe photons. As previously discussed, the occupation probability of a photon in the cavity is always zero, even if the photons pass through the cavity. Here, a puzzling question is raised: How can a photon pass through the cavity if the probability of finding it in the cavity is always zero? This appears to be paradoxical because the photons are transmitted and reflected only via the cavity. The answer to this question is that, in fact, this classical intuition fails. The paradox is based on the semiclassical picture of a sequential process that a photon is first captured in, and then escapes from, the cavity. The reasoning based on this picture would lead to the conclusion that, if the probability of the photon being captured is zero, there would be no chance of transmitting a photon. Interestingly, this semiclassical concept is invalid, and we find a finite probability of finding a photon transmission through the cavity while it is never occupied, even virtually, in the cavity. This is a clear example demonstrating the counter-intuitive nature of quantum theory. Therefore, we can conclude that the measurement-induced dephasing investigated here originates purely from the vacuum fluctuation. The qubit is interacting with the vacuum electric field in the cavity, and this interaction influences the cavity mode. The frequency of the vacuum field depends on the qubit state, thereby transferring the qubit state information into the probe photons.

Conclusion - We predicted that a qubit state is dephased by the zero-point fluctuation of the cavity mode. This vacuumfluctuation-induced dephasing can be verified in a real experiment of a circuit (cavity) QED setup. The vacuum-induced dephasing has a pure quantum mechanical origin which cannot be understood in terms of the photon shot noise. The role of a vacuum as "something" in distinction from "nothing" has been highlighted through various interesting phenomena such as Casimir force, ${ }^{18)}$ Lamb shift, ${ }^{19)}$ and spontaneous emission, ${ }^{20,21)}$ etc. The vacuum-fluctuation-induced dephasing is another intriguing phenomenon manifested by the vacuum electromagnetic field.

This work was supported by the National Research Foundation of Korea under Grant No. 2012R1A1A2003957.

1) N. Bohr, in Quantum Theory and Measurement, ed. J. A. Wheeler and W. H. Zurek (Princeton Univ. Press, Princeton, 1983) Vol. 9, p.9.

2) X. Y. Zou, L. J. Wang and L. Mandel, Phys. Rev. Lett. 67, 318 (1991).

3) M. Brune, E. Hagley, X. Maitre, A. Maali, C. Wunderlich, J. M. Raimond, and S. Haroche, Phys. Rev. Lett. 77, 4887 (1996).

4) E. Buks, R. Schuster, M. Heiblum, D. Mahalu and V. Umansky, Nature 391, 871 (1998).

5) D.-I. Chang, G.-L. Kim, K. Kang, Y. Chung, H. Lee, M. Seo, M. Heiblum, D. Mahalu, and V. Umansky, Nat. Phys. 4, 205 (2008).

6) A. Blais, R.-S. Huang, A. Wallraff, S. M. Girvin, and R. J. Schoelkopf, Phys. Rev. A 69, 062320 (2004).

7) R. J. Schoelkopf and S. M. Girvin, Nature 452, 664 (2008).

8) J. Q. You and F. Nori, Nature 474, 589 (2011).

9) J. Gambetta, A. Blais, D. I. Schuster, A. Wallarff, L. Frunzio, J. Majer, M. H. Devoret, S. M. Girvin, and R. J. Schoelkopf, Phys. Rev. A 74, 042318 (2006).

10) I. Serban, E. Solano and F. K. Wilhelm, Europhys. Lett. 80, 40011 
11) J. Gambetta, A. Blais, M. Boissonneault, A. A. Houck, D. I. Schuster and S. M. Girvin, Phys. Rev. A 77, 012112 (2008).

12) M. Boissonneault, J. M. Gambetta and A. Blais, Phys. Rev. A 79, 013819 (2009).

13) A. P. Sears, A. Petrenko, G. Catelani, L. Sun, H. Paik, G. Kirchmair, L. Frunzio, L. I. Galzman, S. M. Girvin, and R. J. Schoelkopf, Phys. Rev. B 86. 180504(R) (2012).

14) D. I. Schuster, A. Wallraff, A. Blais, L. Frunzio, R.-S. Huang, J. Majer, S. M., and R.J. Schoelkopf, Phys. Rev. Lett. 94. 123602 (2005).

15) P. Bertet, I. Chiorescu, G. Burkard, K. Semba, C. J. P. M. Harmans, D. P. DiVincenzo, and J. E. Mooij, Phys. Rev. Lett. 95, 257002 (2005).

16) E. T. Jaynes and F. W. Cummings, Proc. IEEE 51, 89 (1963)

17) E. M. Purcell, Phys. Rev. 69, 681 (1946).

18) H. B. G. Casimir, Proc. Kon. Ned. Akad. 51, 793 (1948).

19) W. E. Lamb, Jr. and R. C. Retherford, Phys. Rev. 72, 241 (1947).

20) A. Einstein, Phys. Z. 18, 121 (1917).

21) V. Weisskopf and E. Wigner, Z. Phys. 63, 54 (1930).

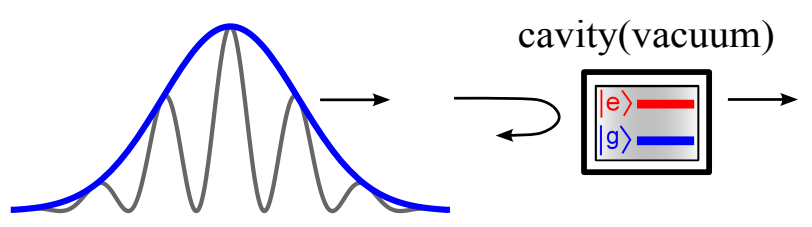

Fig. 1. Schematic diagram of a qubit embedded in a cavity. Single "probe" photons are sent to the cavity, but the cavity is always in the vacuum state during the interaction process. (a)

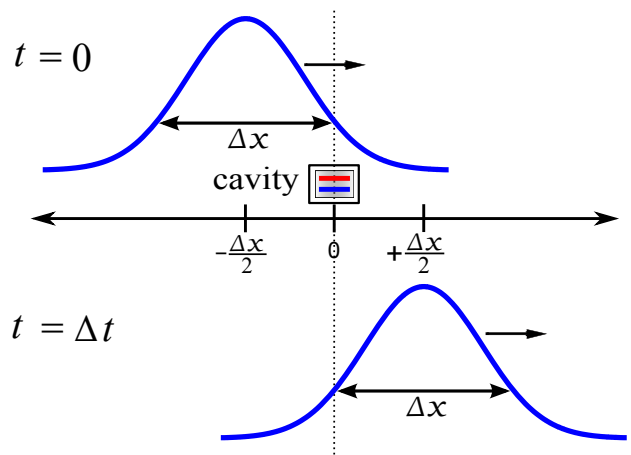

(b)

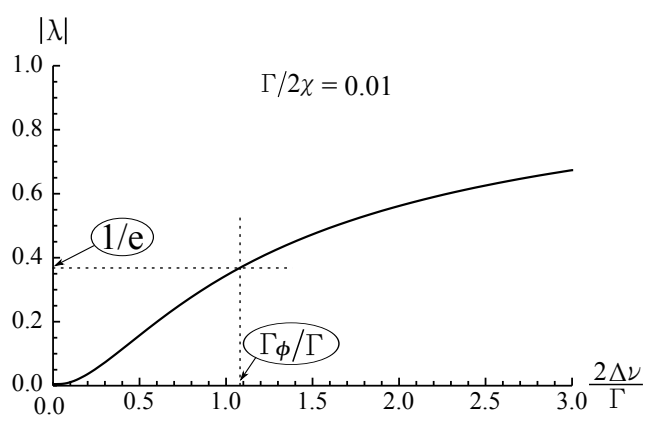

Fig. 2. (a) Illustration of a "measurement" using a single probe photon with the packet size $\Delta x$. The probe photon interacts with the cavity during the time interval of $\Delta t=(2 \Delta v)^{-1}=\Delta x / c$. (b) The coherence factor $(|\lambda|)$ as a function of the frequency dispersion $(\Delta v)$ of the probe photon in a strong measurement limit, $\Gamma / 2 \chi=0.01$. The dephasing time is determined by the travelling time $(\Delta t)$ of the packet time together with the condition $|\lambda|=1 / e$ (see text). 


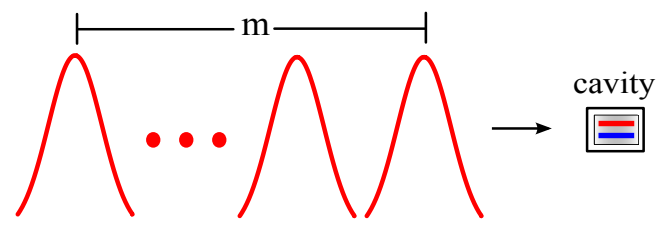

Fig. 3. Continuous weak measurement scheme. The measurement-induced dephasing is determined by a continuous injection of the probe photons, while a single photon dephases the qubit state only very weakly.

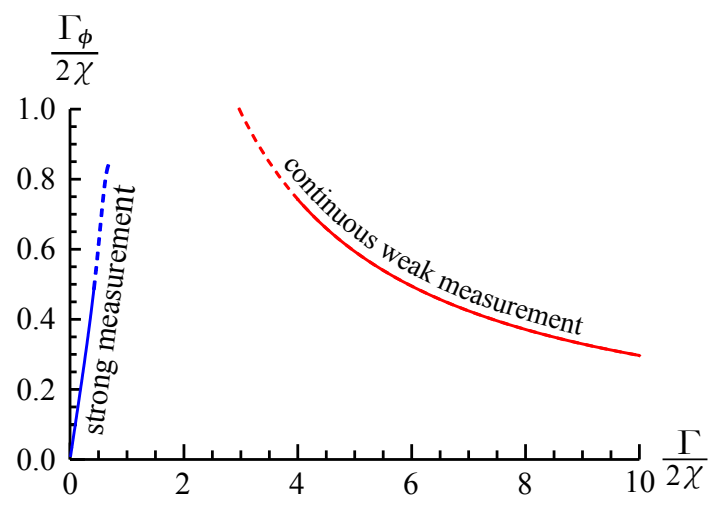

Fig. 4. Numerical result of the vacuum-fluctuation-induced dephasing rate for the strong measurement (blue line), and for the continuous weak measurement (red line) limits, respectively. 